\title{
Induction spectrometry using an ultrafast hollow-cored toroidal-coil (HTC) detector
}

\author{
Yunieski Arbelo ${ }^{1}$ and Davide Bleiner ${ }^{1,2, a)}$ \\ ${ }^{1}$ Swiss Federal Laboratories for Materials Science and Technology (Empa), Überlandstrasse 129, \\ CH-8600 Dübendorf, Switzerland \\ ${ }^{2}$ University of Zürich, Rämistrasse 71, 8006 Zürich, Switzerland
}

(Received 25 September 2016; accepted 22 January 2017; published online 15 February 2017)

\begin{abstract}
Ultrafast photoelectron and photoion spectroscopy (as well as their combination known as "coincidence spectroscopy") utilizes detectors based on different electron multipliers such as microchannel plates or single-channel electron multipliers. These detectors have a few important limitations such as fast-signal distortion (low pass operation), mutually exclusive positive or negative mode, dead time, and requirement of trigger. A high-pass induction detector, based on a hollow-cored toroidal coil, was developed that overcomes the above-mentioned limitations. The frequency-dispersive response and linearity of different configurations were analyzed. It is shown that the response is enhanced for ultrafast electron signals, dependent on construction parameters, thus offering response flexibility by design. Kinetic energy distributions of pseudospark-induced electron pulses are characterized in order to validate the capabilities in real applications. Published by AIP Publishing. [http://dx.doi.org/10.1063/1.4975402]
\end{abstract}

\section{INTRODUCTION}

Different spectrometries allow complementary insights on the chemistry of materials. ${ }^{1-4}$ In general, it utilizes pumpprobe configurations, where an ultrafast pulse (i.e., much shorter that ordinary integration times) excites the target sample and a second pulse (e.g., XUV) probes its atomic/molecular response at varying time delays. ${ }^{5}$ Variations in electron energy distribution can be observed through valence and core level spectra, and mass spectra map the molecular composition. Indeed, coupling electron energy detection with its mass spectrometry conjugate signal leads to coincidence data (PEPICO spectroscopy). ${ }^{6}$

State-of-the-art detectors used in charged particle spectroscopy ${ }^{7}$ are based on different electron multipliers such as microchannel plates (MCP) or single-channel electron multipliers (CEM). The MCP can provide an electron gain from $10^{4}$ to $10^{7}$ and best response times as low as $0.2 \mathrm{~ns},{ }^{8}$ while the CEM allows gains of $10^{8}$ and response times between 10 and 20 ns. $^{9}$

A known drawback of the ordinary detectors is related to distortion of fast signals, due to low-pass operation (Bode diagram). The high-frequency component of a signal bandwidth is responsible for the steep leading edge. If the readout setup cannot preserve the high-frequency components, the output signal typically has a smeared-out profile. This can limit the time-resolution as well as enhance interference and cross-talk.

Ordinary detectors do also require a reliable and jitter-free triggering system, in order to "catch" the important information of an analytical signal. The related instrumentation required to deal with such issues enhances the complexity

\footnotetext{
a)Electronic mail: davide.bleiner@empa.ch
}

and cost of the analytical instrumentation, especially when coincidence photoelectron photoion spectroscopies ${ }^{6}$ are carried out.

Another drawback is that bulk detectors along the beam path stop the incident beam to be measured, e.g., electrons. ${ }^{10}$ This restricts the possibility of online monitoring while processing, which can be advantageous, e.g., in nano-fabrication ${ }^{11}$ and nano-structuring using focused beams, or to study correlated electron/photon bunches, such as in Auger electron spectroscopy (AES). ${ }^{12}$

Typically, in photoelectron time-of-flight (TOF) measurements, one faces the limitation of duty cycle to $10 \%-20 \%$, i.e., modulated sampling in order to avoid electron bunch overlapping or space charge effects, ${ }^{13}$ or detector overload. This restricts the possibility of high duty cycle for improved sensitivity or small sample consumption. Saturation at high electron count rates (e.g., about $\left.10^{5} \mathrm{cps}\right)^{14}$ is also another constraint of operation. For instance, the MCP detector has been used in soft x-ray photoelectron spectroscopy to analyze molecular bonding, ${ }_{5}^{5}$ and limitations due to high number of electrons hitting the MCP, in a time shorter than its response time, were reported.

The aim of this work was to develop a hollow-cored toroidal coil detector (HTC) with high-pass response for ultrafast photoelectron spectroscopy, addressing the abovementioned issues. In order to test the capabilities of the HTC, electron pulses generated on a pseudospark XUV-source ${ }^{15}$ were characterized. Thus, the HTC allows combining the advantage of surface-sensitivity of the XUV photons.

In particular, the HTC was implemented to detect electron signals, however also spectroscopic photoion measurements can be carried out with this detector.

This study is structured as follows: Section II presents the electrical design/theory of the HTC and experimental setups. Section III presents simulated and experimental 
frequency-resolved response of the HTC, measurements of linearity, time-resolved response of the HTC, measurements of XUV-pulses, and detection of pseudospark electron pulses. Conclusions are given in Section IV.

\section{MATERIALS AND METHODS}

\section{A. Electrical design}

The measured currents are passed through the HTC as shown in Fig. 1(a). The rate of change of the input current, $\frac{d I}{d t} \equiv \dot{I}$, induces an image current on the HTC, measured as a voltage, $U_{\text {out }}(\mathrm{t})$, across the resistor $R$. To prevent the influence of stray currents, the HTC is designed with two wire-loops in opposite windings. This allows the cancellation of electromagnetic inputs outside the HTC periphery. The primary loop is made-up of turns of the coil and the secondary loop can be formed by returning the wire through the center of the winding.

The output signal of the HTC must be passed through an integrator circuit to obtain the input current waveform. Otherwise, self-integration techniques can be implemented. ${ }^{16}$

\section{B. Theory of operation}

The response of the HTC was simulated considering the equivalent circuit with a terminating resistance, $\mathrm{R}$, presented in Fig. 1(b). From this circuit the transfer function, ${ }^{17} \mathrm{H}(\mathrm{s})$, can be obtained, which is a function of the output voltage, $U_{\text {out }}(s)$, the input current, $I(s)$, the Laplace variable, $s$, the mutual inductance, $M$, the capacitance of the windings, $C$, the self-inductance, $L$, the resistance of the windings, $r$, and the terminal resistance $R$,

$$
H(s)=\frac{U_{\text {out }}(s)}{I(s)}=\frac{M s}{L C s^{2}+\left(\frac{L}{R}+r C\right) s+\frac{R+r}{R}} .
$$

The mutual inductance of the HTC is calculated as follows:

$$
M=\frac{\mu_{0} N h}{2 \pi} \ln \frac{b}{a},
$$

where $N$ is the number of turns, $\mu_{0}$, the permeability of vacuum, $a$, the inner core diameter, $b$, the external core diameter, and $h$, the core thickness (Fig. 1). $L$ is defined as

$$
L=N M
$$

while $C$ is obtained from

$$
C=\frac{4 \pi^{2} \varepsilon_{0}(b+a)}{\log \left(\frac{b+a}{b-a}\right)}
$$

a)

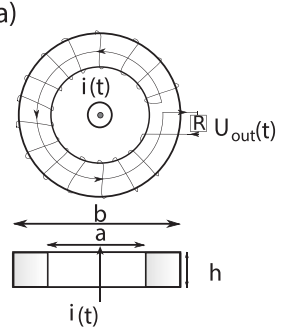

b)

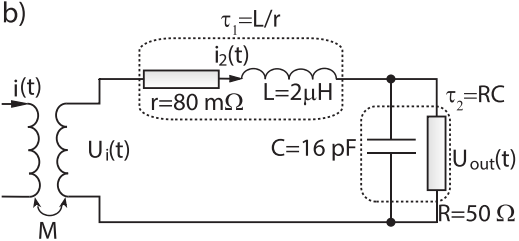

FIG. 1. (a) Schematic of the HTC where the geometric parameters, terminal resistance, $R$, input current, $i(t)$, and output voltage, $U_{\text {out }}(\mathrm{t})$ can be observed. (b) Equivalent circuit of the HTC. where $\varepsilon_{0}$ is the vacuum permittivity. The resistance, $r$, is obtained from

$$
r=\rho \frac{4 l}{\pi d^{2}},
$$

where $l$ and $d$ are the length and diameter of the wire and $\rho$ the density. Considering Eq. (1) and imposing $\tau_{2}<<\tau_{1}$ such that the data readout time constant " $\tau_{2}$ " does not degrade the ultrafast speed of the HTC probe, with time constant " $\tau_{1}$ " (the quantitative validity of this condition is shown in Section III A), the lower $\left(\omega_{l}\right)$ and higher $\left(\omega_{h}\right)$ cutoff frequencies of the HTC can be obtained as follows: ${ }^{17,18}$

$$
\begin{gathered}
\omega_{l}=\frac{R+r}{L+\operatorname{RrC}} \approx \frac{R}{L}, \\
\omega_{h}=\frac{L+\operatorname{RrC}}{L R C} \approx \frac{1}{R C} .
\end{gathered}
$$

Consequently, the bandwidth of the HTC can be obtained as

$$
\Delta \omega=\omega_{h}-\omega_{l} \approx \frac{1}{R C}-\frac{R}{L} .
$$

From Eqs. (3), (4), and (8) one can observe that the operational range of the HTC can be optimized by choosing the geometric parameters, $a, b, h$, the terminal resistance $\mathrm{R}$, and the number of turns N.

Furthermore, for spectroscopic analysis of electron signals, one obtains that the electron kinetic energy, $K$, can be obtained from $K=q V$, where the inductive potential is $V=\mathrm{L} \frac{d I}{d t}$. The inductance $\mathrm{L}$ must be replaced by the mutual inductance M from Eq. (2) and the charge $q=e=1.6 \times 10^{-19} \mathrm{C}$, such that one obtains the expression for the single-energy electron signal

$$
K_{e}=q M \dot{I}_{e}(t) .
$$

Integration over the entire full-energy spectrum gives the electron energy distribution.

For the analysis of ion signals, a mass-to-charge (m/q) relation can be obtained. Considering that the ion kinetic energy $K_{i}$ can be expressed knowing $v_{i}$, i.e., the transition speed of the $i$ th ion, one obtains

$$
K_{i}=\frac{1}{2} m v_{i}^{2}=\frac{1}{2} m\left(\frac{x}{2 \pi / \omega_{i}}\right)^{2},
$$

where $x$ is the flight distance between the ion source and the HTC detector, and recasting the speed as a function of $\omega_{i}$, i.e., the $i$ th ion transition frequency. The latter accounts for the time-of-flight as simple as follows: $T O F=2 \pi / \omega$. Taking into account Eq. (9) (with $K_{i}$ and $q$ the ion charge) one can equate to Eq. (10) to give the mass-to-charge as a function of ion transition frequency, as follows:

$$
(m / q)_{i}=\frac{8 \pi^{2} M}{x^{2} \omega_{i}^{2}} \dot{I}(t) .
$$

The expression gives the mass-to-charge of the $i$ th ion of the MS signal. Integration over the entire ion spectrum gives the full MS spectrum.

The electron or ion current derivative, $\dot{I}(t)$, is experimentally measured and decomposed in frequencies using Fourier transformation as follows:

$$
\dot{I}(t)=\mathcal{F}^{-1}[i \omega \hat{I}(\omega)] .
$$


TABLE I. Electrical and geometric parameters of the studied configurations of the HTC.

\begin{tabular}{lcccc}
\hline \hline & \multicolumn{4}{c}{ HTC configuration } \\
\cline { 2 - 5 } Parameter & A & B & C & D \\
\hline$N(\cdots)$ & 34 & 16 & 34 & 34 \\
$R(\Omega)$ & 50 & 50 & 50 & 50 \\
$h(\mathrm{~mm})$ & 10 & 10 & 10 & 25 \\
$b(\mathrm{~mm})$ & 30 & 30 & 30 & 30 \\
$a(\mathrm{~mm})$ & 15 & 15 & 25 & 15 \\
\hline \hline
\end{tabular}

\section{Experimental set up}

The frequency-resolved response of the four configurations of the HTC presented in Table I were measured using a network analyzer (HP 8753B). In the same way, the linearity and time-resolved response of these configurations of the HTC were obtained using function generators, as shown in Fig. 2. For the linearity measurements, input waveforms with $30 \mathrm{MHz}$ frequency and peak-peak amplitudes that varied between $0.5 \mathrm{~V}$ and $4.0 \mathrm{~V}$ were used, while for the analysis of the time-resolved response, input pulses with duration (FWHM) of $10 \mathrm{~ns}$ and 100 ns were utilized.

The pseudospark-source ${ }^{15}$ used for the generation of electron pulses and XUV-radiation was based on a system of hollow electrodes, filled with working gas (Ar) and directly attached to a storage capacitor bank $(\mathrm{C}=960 \mathrm{nF})$, as shown in Fig. 3. The capacitor is charged until a breakdown voltage ranging between 1.7 and $2.1 \mathrm{kV}$, depending on the working gas type, pressure (0.08-0.12 mbar for Ar), and electrode gap $(\mathrm{d}=6 \mathrm{~mm})$. Following the breakdown, a plasma generated in the hollow cathode shunts the electrode gap, leading to the discharge of the capacitor and generation of a high pulsed current (10-20 kA on $100 \mathrm{~ns}) .^{19,20}$ The magnetic force generated by this current overcomes the thermal pressure and the plasma is compressed in the radial direction, leading to emission of XUV-radiation. In combination with the XUV-emission, electron pulses are also emitted. ${ }^{21}$

In order to estimate the frequency-response required for the HTC to detect XUV-induced photoemission signals, timeresolved measurements of the XUV-pulses emitted from the plasma source were carried out. For these measurements, an

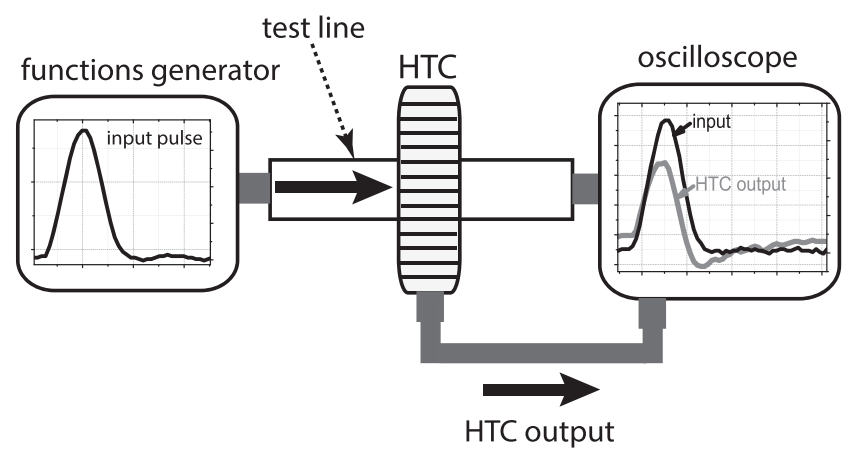

FIG. 2. Experimental setup used to analyze the linearity and time-resolved response of the HTC. The input pulse is passed through the HTC and observed at the oscilloscope together with the response of the HTC.

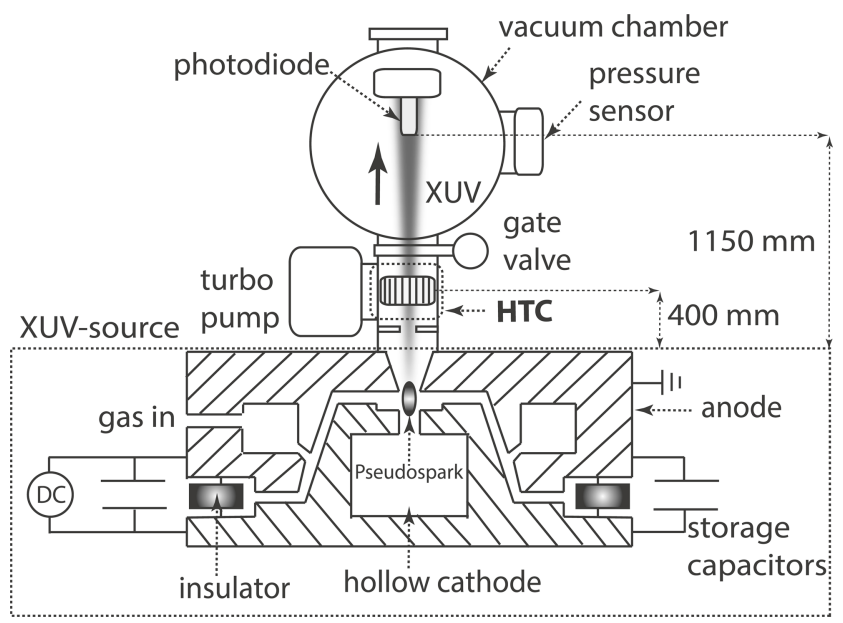

FIG. 3. Experimental setup used for the detection of pseudospark-electron pulses and XUV-radiation. The possibility of monitoring correlated photon beams and electron pulses in the same direction of propagation can be noted.

AXUV-photodiode positioned at $1150 \mathrm{~mm}$ from the plasmasource was used, as shown in Fig. 3. In the same way, measurements of the discharge voltage were realized using a HV probe connected to the cathode.

Measurements of pseudospark-driven electron pulses were performed positioning the HTC at $400 \mathrm{~mm}$ from the source, as shown in Fig. 3. In these measurements, the source was operated with $\mathrm{Ar}$ at different pressures $(0.08 \mathrm{mbar}$ and 0.12 mbar).

\section{RESULTS AND DISCUSSIONS}

\section{A. Characterization of operation}

\section{Parameters Simulation}

As discussed in Sec. II B, the transfer function input to output of the HTC is dependent on the geometry (Fig. 1, parameters $a, b, h)$ and electrical circuit $(R$ and $N)$. In this section this dependence is analyzed quantitatively.

Fig. 4(a) shows the amplitude and phase of the HTC with the geometric inner diameter, $a$, varied in $25 \mathrm{~mm}, 15 \mathrm{~mm}$, and $5 \mathrm{~mm}$. As it can be observed, the peak gain remains the same in the three cases. In the same way, one can observe that a decrease on $a$ induces a decrease on the lower and higher frequencies, $f_{l}$ and $f_{h}$. Moreover, it is noted that the phase changes between 0.8 and -0.8 rad between the lower and higher frequencies for the three configurations of the HTC.

Fig. 4(b) shows the amplitude and phase of the HTC as a function of the geometric thickness, $h$, at $100 \mathrm{~mm}, 10 \mathrm{~mm}$, and $5 \mathrm{~mm}$. As it can be observed, the peak gain and the higher frequency remain unaffected with the variation of $h$. In contrast, it is observed that an increase on $h$ induces a decrease in the lower frequency, $f_{l}$. This behavior can be explained from Eqs. (2), (3), and (6), since as $h$ is increased, the self-inductance of the HTC is increased and the lower frequency is decreased. In particular, from this analysis the possibility of increasing the bandwidth of the HTC by incrementing $h$ can be noted. On the other hand, the phase changes from around $0.8 \mathrm{rad}$ to -0.8 rad between the lower and higher frequencies for the three configurations of the HTC. 

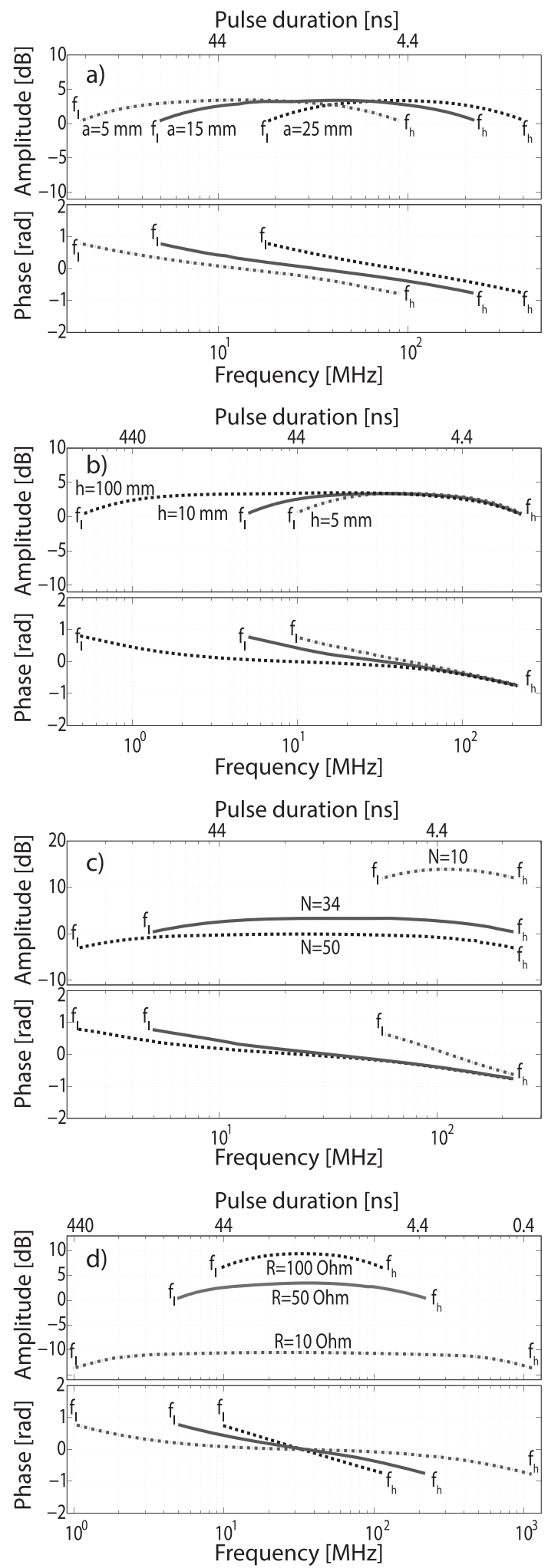

FIG. 4. Simulated frequency-resolved response of the HTC, (a) changing the geometric inner diameter $a$, (b) changing the geometric thickness $h$, (c) changing the number of turns $N$, and (d) changing the terminal resistance $R$.

Fig. 4(c) shows the amplitude and phase of the HTC with the number of turns, $N$, at 50,34, and 10 turns. The higher frequency, $f_{h}$, remains the same in the three cases. On the other hand, it can be observed that a decrease in $N$ induces an increase in the peak gain and in the lower frequency, $f_{l}$.
In particular this dependence of $f_{l}$ can be explained as in Fig. 4(b), from Eqs. (2), (3), and (6), as $N$ is decreased, the self-inductance of the HTC is decreased increasing the lower frequency. It should be noted that the self-inductance is dependent on the square of the number of turns. Besides, the phase changes between the lower and higher frequencies from around $0.8 \mathrm{rad}$ to $-0.8 \mathrm{rad}$ for the configurations with $N=34$ and $N=50$ and from around $0.6 \mathrm{rad}$ to $-0.6 \mathrm{rad}$ for the HTC with $N=10$.

Fig. 4(d) shows the amplitude and phase of the HTC with the terminal resistance, $R$, at $100 \Omega, 50 \Omega$, and $10 \Omega$. In this case it can be observed that an increase of $R$ increases the peak gain but reduces the bandwidth of the HTC. Namely, the lower frequency is increased while the higher frequency is decreased. This can be explained from Eqs. (6)-(8). On the other hand, the phase changes between the lower and higher frequencies from around $0.8 \mathrm{rad}$ to $-0.8 \mathrm{rad}$ for the configurations with $R=10 \Omega$ and $R=50 \Omega$ and from around $0.7 \mathrm{rad}$ to $-0.7 \mathrm{rad}$ for the HTC with $R=100 \Omega$.

\section{Experimental response}

The frequency-resolved response of four configurations were experimentally obtained in order to test the simulated parametric dependence, presented in Sec. III A 1, and to optimize the response of the detector around $6 \mathrm{MHz}$, as required for the detection of our photoemission "test case."

In Fig. 5(a) the Bode diagram shows the experimental frequency-resolved response of the HTC with the configuration $N=34, R=50 \Omega, r=80 \mathrm{~m} \Omega, h=10 \mathrm{~mm}, b=30 \mathrm{~mm}$, and $a=15 \mathrm{~mm}$. When these parameters are used to test the condition $\frac{\tau_{1}}{\tau_{2}} \gg 1$ presented in Section II B, a value of $\tau_{1}$ of $2.1 \cdot 10^{4} \mathrm{~ns}$ is obtained while for $\tau_{2}$ a value of about $0.8 \mathrm{~ns}$ is estimated, giving $\frac{\tau_{1}}{\tau_{2}}>10^{4}$.

For this configuration of the detector, the lower frequency is obtained at $4 \mathrm{MHz}$. In the same way, a resonance in the response of the HTC at $96 \mathrm{MHz}$ is observed. These resonances are originated due to self-inductance and capacitance distributed between the windings of the HTC.

Fig. 5(b) shows the experimental frequency-resolved response of the HTC reducing $N$ and keeping the parameters $R$, $h, b$, and $a$ as in the configuration (A). An increase in the lower frequency and gain is observed as the parameter $N$ is reduced, which is in agreement with the parametric study presented in Fig. 4(c). The lower frequency is obtained in around $11 \mathrm{MHz}$.

Fig. 5(c) shows the experimental frequency-resolved response of the HTC increasing a and keeping the parameters $N, R, h$, and $b$ as in configuration (A). The gain remains the same while the lower frequency is increased. This response is in agreement with the parametric analysis presented in Fig. 4(a). In this case, the experimental lower frequency is obtained in around $8 \mathrm{MHz}$.

Fig. 5(d) shows the experimental frequency-resolved response of the HTC increasing $h$ and keeping the parameters $N, R, b$, and $a$ as in the configuration (A). The same gain is obtained while the lower frequency is decreased, as similarly presented in Fig. 4(b). The experimental lower frequency is obtained in around $3 \mathrm{MHz}$. In the same way, a resonance in the response of the HTC is observed at $60 \mathrm{MHz}$. 

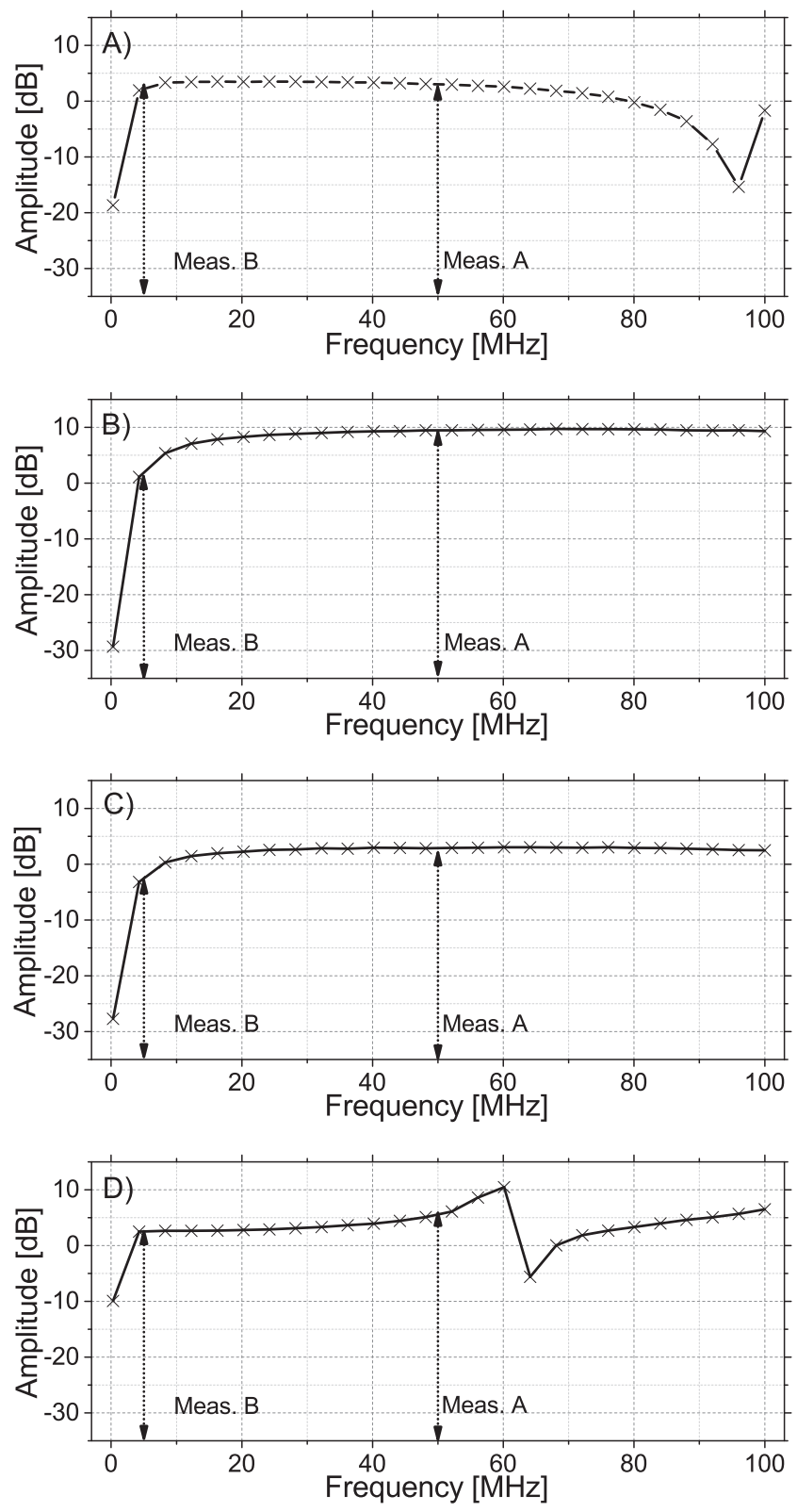

FIG. 5. Experimental frequency-resolved response of the HTC with different configurations: (A) $\mathrm{N}=34, \mathrm{R}=50 \Omega, \mathrm{h}=10 \mathrm{~mm}, \mathrm{~b}=30 \mathrm{~mm}$, and $\mathrm{a}=15 \mathrm{~mm}$. (B) $\mathrm{N}=16, \mathrm{R}=50 \Omega, \mathrm{h}=10 \mathrm{~mm}, \mathrm{~b}=30 \mathrm{~mm}$, and $\mathrm{a}=15 \mathrm{~mm}$. (C) $\mathrm{N}=34$, $\mathrm{R}=50 \Omega, \mathrm{h}=10 \mathrm{~mm}, \mathrm{~b}=30 \mathrm{~mm}$, and $\mathrm{a}=25 \mathrm{~mm}$. (D) $\mathrm{N}=34, \mathrm{R}=50 \Omega, \mathrm{h}=25$ $\mathrm{mm}, \mathrm{b}=30 \mathrm{~mm}$, and $\mathrm{a}=15 \mathrm{~mm}$. The frequency values $\mathrm{A}$ and $\mathrm{B}$ correspond to pulses with duration of 10 and $100 \mathrm{~ns}$ as presented in Fig. 7.

Considering the stability on the response of the four HTCs studied around the frequency of $6 \mathrm{MHz}$, the configuration (A) can be used to perform measurements of XUV-induced photoemission.

\section{Linearity of the HTC}

Fig. 6 shows the measured linearity of the four configurations of the HTC (Table I). A linear response for the four configurations is observed. For the configurations (A), (C), and (D), the same input-output dependence is obtained. On the other hand, a higher response (around factor of 2) is observed for the configuration (B) of the HTC, where the number of turns $(N)$ was reduced (about factor of 2). This is in agreement with

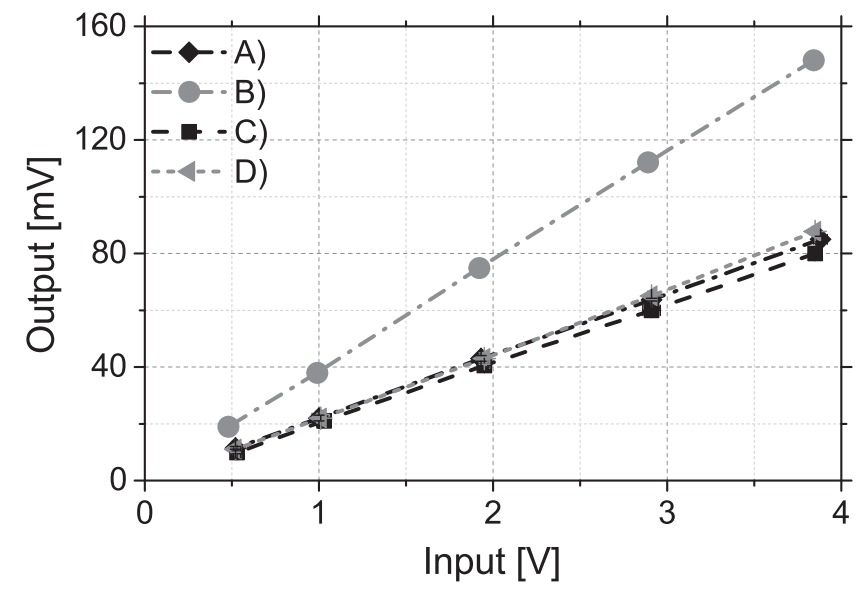

FIG. 6. Measured linearity of four configurations of the HTC: (A) $N=34$, $\mathrm{R}=50 \Omega, \mathrm{h}=10 \mathrm{~mm}, \mathrm{~b}=30 \mathrm{~mm}$, and $\mathrm{a}=15 \mathrm{~mm}$. (B) $\mathrm{N}=16, \mathrm{R}=50 \Omega, \mathrm{h}=10$ $\mathrm{mm}, \mathrm{b}=30 \mathrm{~mm}$, and $\mathrm{a}=15 \mathrm{~mm}$. (C) $\mathrm{N}=34, \mathrm{R}=50 \Omega, \mathrm{h}=10 \mathrm{~mm}, \mathrm{~b}=30 \mathrm{~mm}$, and $\mathrm{a}=25 \mathrm{~mm}$. (D) $\mathrm{N}=34, \mathrm{R}=50 \Omega, \mathrm{h}=25 \mathrm{~mm}, \mathrm{~b}=30 \mathrm{~mm}$, and $\mathrm{a}=15 \mathrm{~mm}$. The plot shows a limited range due to limitations in instrumentation, however, no saturation is expected (unlimited dynamic range).

the fact that the sensitivity of the HTC is inversely proportional to $N$. In particular, for the HTC an unlimited dynamic range is expected.

\section{Time-resolved response of the HTC}

Fig. 7 shows the time-resolved response of the configurations (A) and (B) of the HTC for input pulses with duration (FWHM) of $10 \mathrm{~ns}$ (a) and $100 \mathrm{~ns}$ (b). In both panels, a differential response for the two configurations of the HTC is observed. This is due to the fact, that the amplitude of HTC (voltage induced in the coil) is proportional to the differential of the current.

\section{B. Application to XUV photoemission spectroscopy}

Fig. 8 shows preliminary measurements of the XUVpulses emitted from the pseudospark-source and the related discharge voltage waveform. The XUV emission is observed to reach the maximum $358 \mathrm{~ns}$ after the discharge is triggered. In the same way, it can be observed that the XUV pulse has a duration of about $170 \mathrm{~ns}$ (FWHM), therefore, the HTC can provide response at $6 \mathrm{MHz}$.

Figs. 9(a) and 9(b) show time-resolved HTC measurements of the pseudospark electron pulses, as shown in Fig. 3. The source is operated with $\mathrm{Ar}$ at pressures of 0.08 mbar (a) and 0.12 mbar (b). In both panels, several electron peaks correlated to the oscillations of the discharge voltage are observed. In particular, a differential response of the HTC to the electron peaks is observed, as similarly shown in Fig. 7. At the pressure of 0.08 mbar (a) the main electron peak is observed $236 \mathrm{~ns}$ after the discharge is triggered, while for the pressure of $0.12 \mathrm{mbar}$ (b) this value is a factor of 3.3 higher. On the other hand, it can be observed that for the higher pressure case (b), the discharge voltage obtained $(1.7 \mathrm{kV})$ is lower by a factor of 1.2 . This is explained by the increase of the working gas ionization with the decrease of the electron mean free path due to the increase of the gas pressure. 

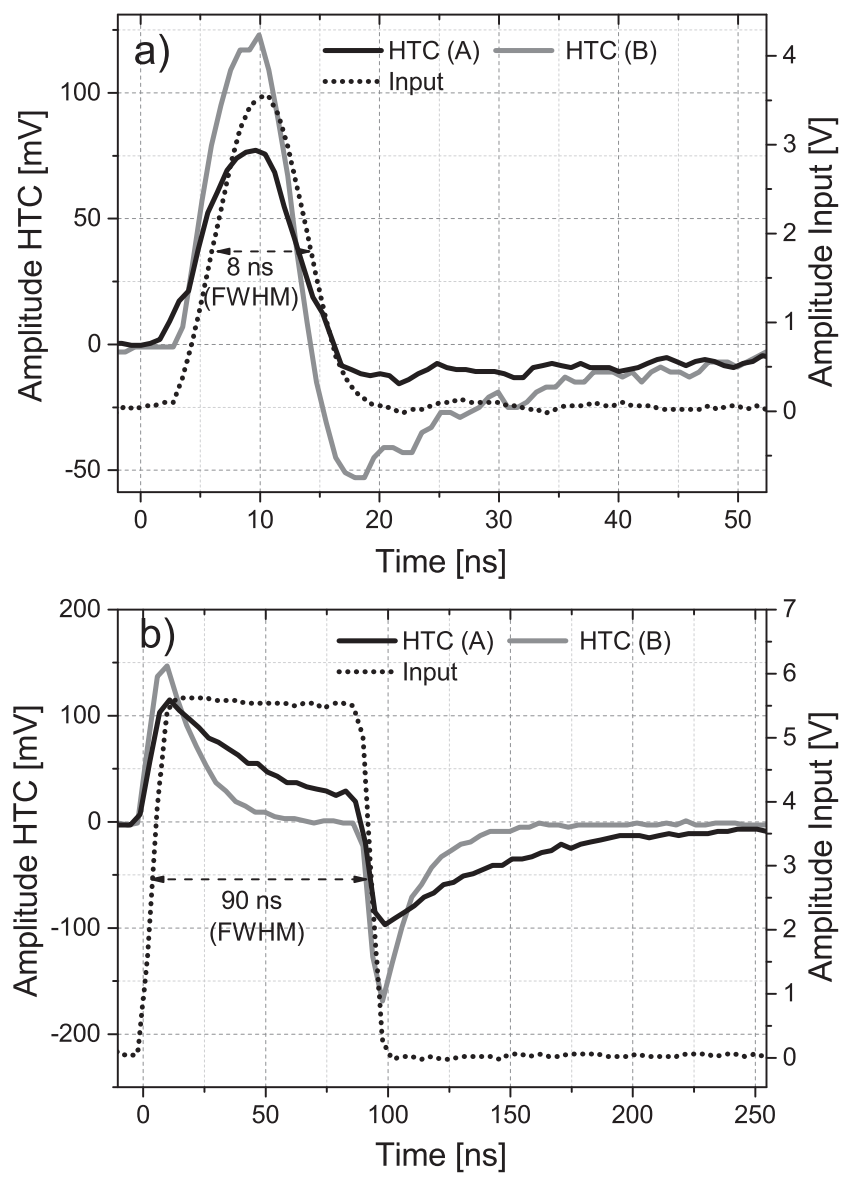

FIG. 7. Time-resolved response of the HTC with the configurations: (A) $(\mathrm{N}=34, \mathrm{R}=50 \Omega, \mathrm{h}=10 \mathrm{~mm}, \mathrm{~b}=30 \mathrm{~mm}$, and $\mathrm{a}=15 \mathrm{~mm})$ and $(\mathrm{B})(\mathrm{N}=16$, $\mathrm{R}=50 \Omega, \mathrm{h}=10 \mathrm{~mm}, \mathrm{~b}=30 \mathrm{~mm}$, and $\mathrm{a}=15 \mathrm{~mm}$ ) for input pulses with duration of $10 \mathrm{~ns}$ (a) and $100 \mathrm{~ns}$ (b). It can be noted that although the input pulse durations were settled at 10 and $100 \mathrm{~ns}$, the measured values were 8 and 90 ns. This was due to limitations of the function generator settings.

Figs. 9(c) and 9(d) show kinetic energy distributions as obtained from the fast Fourier transformation of the pseudospark electron signal presented in Figs. 9(a) and 9(b). In both panels, the electron distributions show the maximum at low energies (below $20 \mathrm{eV}$ ). In particular, for the lower pressure case (c), higher electron kinetic energies are observed related to the higher discharge voltage obtained.

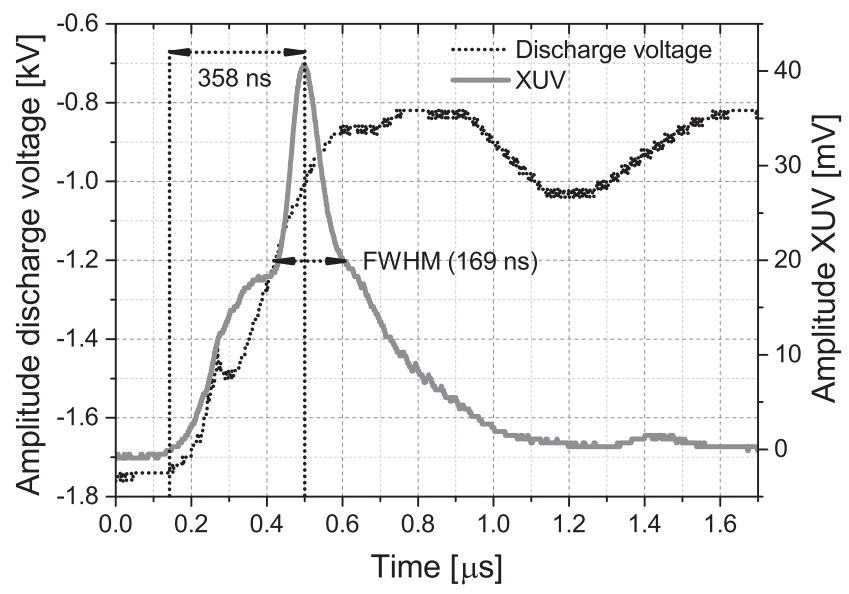

FIG. 8. Time-resolved measurements of the XUV-pulse and related discharge voltage acquired with the photodiode and HV-probe.
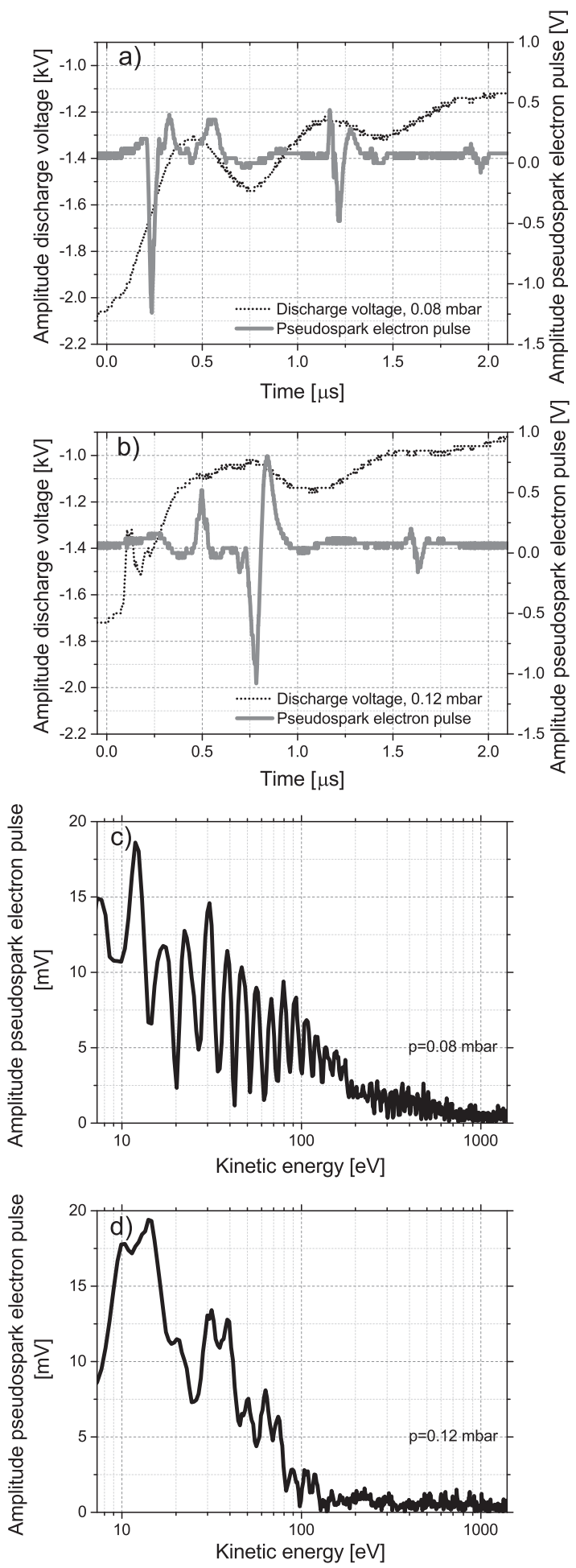

FIG. 9. Pseudospark electron pulse and discharge voltage measured when the source is operated with $\mathrm{Ar}$ at (a) 0.08 mbar and (b) 0.12 mbar. ((c) and (d)) Electron kinetic energy distributions obtained from the electron signals presented in (a) and (b).

\section{CONCLUSIONS}

A hollow toroidal coil (HTC) detector has been developed to perform ultrafast photocoincidence spectroscopy. It was shown that the response of the HTC is dependent on geometric and electrical parameters, which allows for bandwidth selectivity up to the ultrafast domain. An increase in the thickness of the core, $h$, of the HTC was observed to increase 
the bandwidth without affecting the gain. Similarly, it was observed that an increase in the terminal resistance, $R$, increases the gain but reduces the bandwidth.

Overall, the HTC detector has demonstrated overcoming a number of drawbacks for ordinary electron multipliers. The HTC is not affected by electron or ion bunch overlapping as in TOF-MS since it measures in Fourier space. Similarly, it allows measurements of fast signals without distortion, does not suffer saturation, and it can allow unlimited dynamic range. Moreover, it enables online monitoring of correlated photon/electron pulses in the same direction of propagation without pulse stopping, while it does not require demanding triggering systems. In addition, a single HTC can be used to measure in positive/negative mode, replacing the use of several electron multipliers as required for PEPICO. Further work is dedicated to implement this technology for $100 \%$ duty cycle TOF. The HTC detector will enable new kind of optical and mass spectrometries, here termed induction spectrometry.

\section{ACKNOWLEDGMENTS}

The present work was supported by Gebert Rüf Stiftung. The authors are grateful to Andreas Kündig from the Swiss Federal Laboratories for Materials Science and Technology (Empa). The detector is patent pending (Application No./Patent No. 15196565.4-1803).

${ }^{1}$ L. N. Glandorf, M. Scheer, D. A. Samuels, A. M. Mulhisen, E. R. Grant, X. Yang, V. M. Bierbaum, and S. R. Leone, Phys. Rev. Lett. 87, 193002 (2001).

${ }^{2}$ L. Armelao, D. Bleiner, V. Di Noto, S. Gross, C. Sada, U. Schubert, E. Tondello, H. Vonmont, and A. Zattin, Appl. Surf. Sci. 249, 277-294 (2005).
${ }^{3}$ D. Bleiner, P. Lienemann, and H. Vonmont, Talanta 65, 1286-1294 (2005).

${ }^{4}$ D. Bleiner, P. Lienemann, A. Ulrich, H. Vonmont, and A. Wichser, J. Anal. At. Spectrom. 18, 1146-1153 (2003).

${ }^{5}$ L. N. Glandorf, "Time resolved photoelectron spectroscopy with ultrafast soft x-ray light," Ph.D. thesis, University of Colorado, 2001.

${ }^{6}$ G. A. Garcia, H. Soldi-Lose, and L. Nahon, Rev. Sci. Instrum. 80, 023102 (2009).

${ }^{7}$ N. Kamakura, Y. Takata, T. Tokushima, Y. Harada, A. Chainani, K. Kobayashi, and S. Shin, Phys. Rev. B 74, 045127 (2006).

8 Photonis, "Gen2 ultrafast tof detector," www.photonis.com, 2015.

${ }^{9}$ M. J. Kristo and C. G. Enke, Channeltron Electron Multiplier Handbook for Mass Spectrometry Applications (Galileo Electro-Optics Corporation, Sturbridge, 1991).

${ }^{10}$ J. L. Wiza, Nucl. Instrum. Methods 162, 587-601 (1979).

${ }^{11}$ J. Gierak et al., Microelectron. Eng. 57, 865-875 (2001).

${ }^{12}$ A. Gaucher, E. Martinez, J. Baylet, and C. Cardinaud, J. Electron. Mater. 43, 1255-62 (2014).

${ }^{13}$ T. Gießel, D. Bröcker, P. Schmidt, and W. Widdra, Rev. Sci. Instrum. 74, 4620-4624 (2003).

${ }^{14}$ T. Kawauchi, M. Matsumoto, K. Fukutani, T. Okano, S. Kishimoto, X. Zhang, and Y. Yoda, Rev. Sci. Instrum. 78, 013303 (2007).

${ }^{15}$ Y. Arbelo, F. Barbato, and D. Bleiner, "He-doped pseudospark as a home-lab xuv source beyond the beamtime bottleneck," Plasma Sources Sci. Technol. (published online).

${ }^{16}$ M. Argueso, G. Robles, and J. Sanz, Rev. Sci. Instrum. 76, 065107 (2005).

${ }^{17}$ L. Rong, L. Ji-sheng, and F. Yang-yu, Prz. Elektrotechniczny 89, 115-119 (2013).

${ }^{18}$ W. Chen, C. Yao, P. Chen, C. Sun, L. Du, and R. Liao, IEEE Trans. Power Delivery 23, 355-360 (2008).

${ }^{19}$ K. Bergmann, G. Schriever, O. Rosier, M. Muller, W. Neff, and R. Lebert, Appl. Opt. 38, 5413-5417 (1999).

${ }^{20}$ K. Bergmann, F. Küpper, and M. Benk, J. Appl. Phys. 103, 123304 (2008).

${ }^{21} \mathrm{~J} . \mathrm{Hu}$ and J. L. Rovey, "Experimental investigations of high voltage pulsed pseudospark discharge and intense electron beams," in 50th AIAA Aerospace Sciences Meeting including the New Horizons Forum and Aerospace Exposition (American Institute of Aeronautics and Astronautics, 2012), pp. $1-12$. 